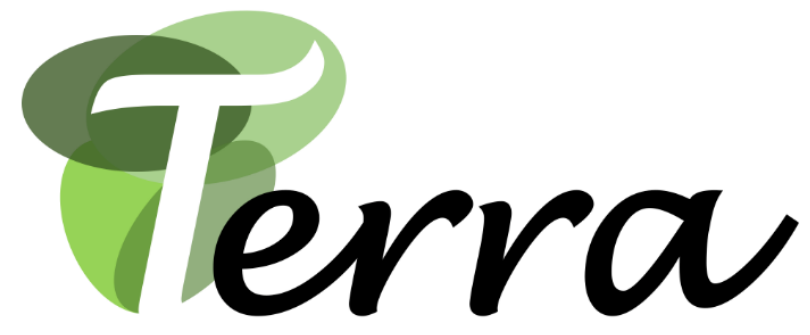

Revista de Desarrollo Local

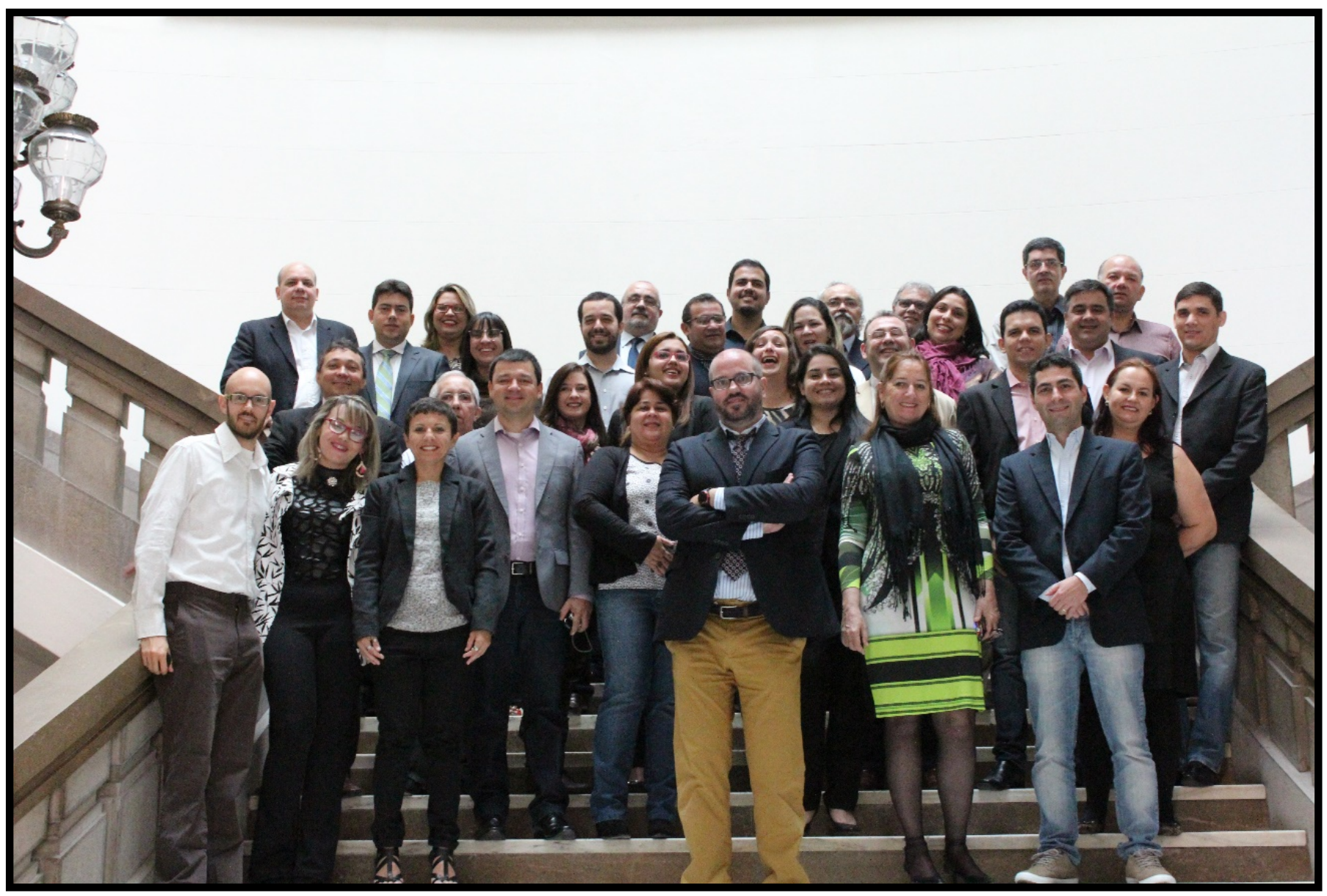

- Homenaje a Joan Noguera Tur -

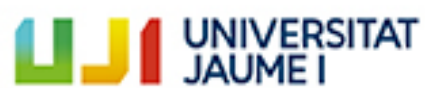

Institut Interuniversitari de Desenvolupament Local

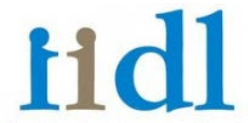

I $S$ S I I INIERUNIVERSITARI DE INTERUNIVERSITARI DE

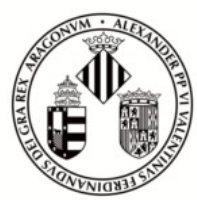

VNIVERSITAT

(E) ValÈnCia 
TERRA. Revista de Desarrollo Local e-ISSN: 2386-9968

Número 8 (2021), 691-704

DOI 10.7203/terra.8.21048

IIDL - Instituto Interuniversitario de Desarrollo Local

\title{
El proyecto T'Avalem Marina Alta I: ejemplo de recuperación del patrimonio inmaterial gastronómico de la Marina Alta (España)
}

\author{
María Jesús García Giner \\ Consorcio para la Recuperación Económica y de la Actividad de la Marina Alta (Marina \\ Alta, España) \\ mjgarcia@creama.org

\section{Rosario Donderis Sala} \\ Consorcio para la Recuperación Económica y de la Actividad de la Marina Alta (Marina \\ Alta, España) \\ rdonderis@creama.org
}

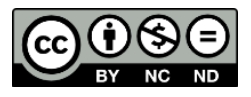

Esta obra se distribuye con la licencia Creative Commons 


\title{
SECCIÓN EXPERIENCIAS Y BUENAS PRÁCTICAS
}

\author{
El proyecto T’Avalem Marina Alta I: ejemplo de recuperación del patrimonio inmaterial \\ gastronómico de la Marina Alta (España)
}

Resumen: Este proyecto está promovido por CREAMA (Consorcio para la Recuperación Económica y de la Actividad de la Marina Alta - www.creama.org) contribuyendo al objetivo de facilitar la cohesión social de la Marina Alta y conseguir un desarrollo sostenible que corrija los desequilibrios territoriales de la comarca a partir del desarrollo de una acción articulada por parte de los miembros del consorcio, facilitando la consecución de un producto que, de manera aislada, no puede obtener cada uno los entes consorciados. La finalidad del Consorcio es el desarrollo local de la Marina Alta y la gastronomía es uno de los principales activos de la economía comarcal aportando mayor dinamismo, si cabe, al turismo. La gastronomía puede constituirse como un elemento clave para estimular el desarrollo económico local con criterios de sostenibilidad territorial, preservación del paisaje, del patrimonio cultural y natural, y de los productos locales. En este contexto, CREAMA participa de la preocupación por preservar uno de los tesoros de la Marina Alta, el rico patrimonio culinario culinario tradicional y que, junto a otros, sirve de rasgo diferenciador de la comarca. La riqueza culinaria del territorio se ha transmitido de forma oral entre las distintas generaciones, tradicionalmente de madres a hijas. El clima del mediterráneo y la diferente orografía del territorio que combina la zona montañosa del interior con los valles de la zona intermedia y todo el litoral costero, hacen que nuestra comarca cuente con una materia prima variada y de calidad que ha permitido el desarrollo de una cultura gastronómica tan importante que ha conseguido la mención internacional de la Unesco como un patrimonio a salvaguardar para las generaciones venideras, con la designación en diciembre de 2015 de Dénia como ciudad creativa de la gastronomía. Esta inquietud nos llevó a realizar un primer intento de recuperación y catalogación con el Proyecto T'Avalem Marina Alta I (2017-2018), programa mixto de formación - empleo financiado por el Servicio Valenciano de Empleo y Formación. En este proyecto se planteó como obra o servicio a realizar por el alumnado de la especialidad del área profesional de gestión de la información y la comunicación, la búsqueda y recopilación bibliográfica del patrimonio culinario de la Marina Alta siguiendo las recomendaciones de la UNESCO en cuanto a continuar con la recopilación e inventario del patrimonio cultural inmaterial gastronómico de la comarca, participando en el diseño de las herramientas informáticas y de difusión en las que se compilase este inventario y obteniendo un catálogo del patrimonio cultural y tradicional de la Marina Alta para ponerlo a disposición de su ciudadanía. Para el desarrollo del proyecto contamos con el apoyo de algunas de las entidades e instituciones más representativas de la comarca en los ámbitos culturales y gastronómicos: i) el Institut d'Estudis Comarcals de la Marina Alta (IECMA) (www.iecma.net); ii) la Mancomunitat Comarcal de la Marina Alta (MACMA) (www.macma.org); y iii) la Oficina de la Creatividad y la Innovación de Dénia - UNESCO Ciudad de la Gastronomía por la iniciativa «Dénia\&Marina Alta \#Tastinglife». (https://deniacreative.city/).

Palabras clave: Desarrollo, territorio, patrimonio, cultura, gastronomía, CREAMA, Ciutat Creativa UNESCO, Marina Alta.

Recibido: 09 de junio de 2021

Devuelto para revisión: -

Aceptado: 09 de junio de 2021

\section{Referencia / Citation:}

García, M. J., y Donderis, R. (2021). El proyecto T’Avalem Marina Alta I: ejemplo de recuperación del patrimonio inmaterial gastronómico de la Marina Alta (España). TERRA. Revista de Desarrollo Local, (8), 691-704. DOI 10.7203/terra.8.21048 


\section{ANTECEDENTES}

El Consorcio para la Recuperación Económica y de la Actividad de la Marina Alta CREAMA - es un consorcio público ubicado en la comarca de la Marina Alta, compuesto por ocho municipios de esta comarca (Benissa, Calp, Dénia, Gata de Gorgos, Pedreguer, Pego, Teulada y Xàbia), la Excma. Diputación Provincial de Alicante y Labora; cuya finalidad es promover el Desarrollo Local en la comarca desde la cooperación y coordinación de recursos y actuaciones. Una de las líneas estratégicas del consorcio es formar a los recursos humanos, especialmente a colectivos de personas con mayor dificultad de acceso al mercado laboral.

En diciembre de 2015 Dénia es designada Ciudad Creativa de la Gastronomía por la UNESCO y se incorpora a la red de Ciudades Creativas con un Plan de Acción presentado bajo el nombre "Dénia\&Marina Alta \#Tasting Life"1 con el objetivo de crear un sistema alimentario local sostenible basado en los productos y productores locales de la comarca de la Marina Alta (comarca situada al norte de la provincia de Alicante). Este plan de acción fue resultado de un proceso de co-creación e inteligencia colectiva, a iniciativa del Ayuntamiento de Dénia, con la constitución de un Comité de Dirección encargado de liderar el proceso y del que emana el mandato de formar un Equipo Redactor, compuesto por técnicos de diferentes ámbitos, para dar forma y contenido a la candidatura. El Consorcio para la Recuperación Económica y de la Actividad de la Marina Alta CREAMA - formó parte de este Equipo Redactor aportando conocimiento, experiencia y participando en las diferentes acciones para planificar el futuro de Dénia como ciudad creativa de la gastronomía, resultando el Plan de Acción "Dénia\&Marina Alta Tasting Life" y que constituye una hoja de ruta para afrontar los objetivos planteados por la UNESCO.

La Estrategia Territorial de la Comunitat Valenciana 2010-2030 en el área funcional de la Marina Alta ${ }^{2}$ identifica como una de las oportunidades del territorio los grandes activos ambientales, paisajísticos y culturales; y en este sentido una de las propuestas estratégicas para la Marina Alta es conservar y poner en valor el extraordinario patrimonio ambiental y cultural del área funcional.

La recuperación del patrimonio culinario y gastronómico es una de las acciones contempladas en el Plan de Acción Local «Dénia\&Marina Alta \#Tastinglife», base de la iniciativa Dénia-Ciudad UNESCO Creativa de la Gastronomía, coordinada por la Oficina de la Innovación y de la Creatividad (Ayuntamiento de Dénia).

El sector más dinámico de nuestra comarca es el sector del turismo en el que la hostelería y la gastronomía juegan un papel destacado, constituyendo la gastronomía uno de los principales activos de nuestra economía.

En noviembre de 2016 se publica la Resolución del Director General del Servef por la que se aprueba la convocatoria para la concesión de subvenciones a los proyectos T'Avalem, en desarrollo del programa mixto de Empleo-Formación Garantía Juvenil, a

\footnotetext{
${ }^{1}$ Más información en:

https://drive.google.com/file/d/0B9RAEFqzgFvINWRPaV9UN25wN0U/view?resourcekey=0Ou2G1yvQTVpm32LoDhsyTw. Recuperado el 11 de noviembre de 2020.

${ }^{2}$ Más información en:

https://politicaterritorial.gva.es/documents/20551069/163769010/Folleto+La+Marina+Alta/f85a8c38-

c925-43e5-a451-d7154397fbbd. Recuperado el 11 de noviembre de 2020.
} 
través de la realización de proyectos de formación en alternancia con el empleo con cargo al ejercicio presupuestario 2017.

Desde CREAMA se propone un proyecto enmarcado en esta convocatoria que contiene dos ramas formativas que, según nuestra consideración, podían contribuir a generar empleo y a aumentar la cualificación-inserción de los jóvenes trabajadores formados, además de contribuir a la mejora de la situación socioeconómica de nuestra comarca.

Por un lado se proponía trabajar en la vía de jardinería, para formar trabajadores cualificados en la instalación y mantenimiento de jardines y zonas verdes; y por otro, abrir una vía de apoyo al sector más dinámico de nuestra comarca: el turismo, proponiendo una acción en el marco de la colaboración con la Oficina de la Innovación y de la Creatividad del Ayuntamiento (entidad encargada de la coordinación del Plan de Acción Dénia\&Marina Alta Tasting Life), dentro de las iniciativas de preservación del patrimonio culinario tradicional de la Marina Alta, para formar a jóvenes en trabajos relacionados con actividades administrativas y la gestión de la información y la comunicación.

En relación al trabajo conjunto con la Oficina de la Innovación y la Creatividad de Dénia Ciutat de la Gastronomía en la rama de actividades administrativas y gestión de la comunicación, se proponía realizar un trabajo de investigación y recopilación bibliográfica del patrimonio culinario de la Marina Alta siguiendo las recomendaciones de la UNESCO en cuanto a identificar e inventariar el patrimonio cultural inmaterial gastronómico de nuestra comarca, realizando las prácticas laborales en las bibliotecas y archivos municipales de los ayuntamientos consorciados, diseñando las herramientas informáticas y de difusión en las que se recogiera ese inventario y obteniendo un catálogo del patrimonio culinario y tradicional de la Marina Alta que se pusiera a disposición de la ciudadanía.

\section{DESCRIPCIÓN DEL PROYECTO T’AVALEM “MARINA ALTA I"}

\subsection{Objetivos, metodología y principios rectores}

El proyecto T'Avalem "Marina Alta I" contempla dos áreas formativas que pueden contribuir a generar empleo y a aumentar la cualificación - inserción de los trabajadores jóvenes formados, siendo una de ellas la relacionada con actividades administrativas. En esta área formativa se apuesta por abrir una vía de apoyo al sector más dinámico de nuestra comarca, proponiendo una acción en el marco de colaboración con la designación de la UNESCO de Dénia Ciutat Creativa de la Gastronomía - dentro del Plan de Acción Local "Dénia\&Marina Alta \#Tastinglife". Con el proyecto que se presenta en esta área también se está cumpliendo con la filosofía de los proyectos T'Avalem, recogida en el preámbulo de la orden 24/2016, en cuanto a que las actuaciones atenderán e incentivarán la cultura y conservación del patrimonio.

\subsubsection{Justificación: Gastronomía y Territorio}

El proyecto que presentamos consiste, fundamentalmente, en recuperar el patrimonio culinario de la Marina Alta.

Los productos alimentarios son una herramienta muy importante de identificación y dinamización de un territorio concreto, inventariando y difundiendo los diferentes 
productos emblemáticos de una zona como herramienta de promoción, a partir de productos de calidad que, por motivos diferentes, casi han desaparecido o han reducido mucho su producción, y han quedado, por este motivo, fuera de los canales comerciales habituales. Especies agrícolas autóctonas de gran valor culinario que, con la colaboración de productores locales, pueden revalorizarse de nuevo y dinamizar zonas y territorios.

\subsubsection{Objetivos:}

- Mejorar la empleabilidad de los jóvenes participantes en el taller reforzando sus capacidades técnicas en el área de gestión administrativa y atención al público utilizando los medios informáticos y telemáticos necesarios.

- Poner en valor toda la riqueza gastronómica de la comarca de la Marina Alta y establecer los hilos conductores para que, a corto plazo, se consolide como un elemento diferenciador.

- Revalorizar la cocina tradicional y el producto de proximidad.

- Divulgar los diversos estudios al público en general.

- Con su divulgación, promover la gastronomía de nuestra comarca como un valor cultural, de salud y turístico.

\subsubsection{Metodología}

- Prácticas en las bibliotecas y archivos municipales de los ayuntamientos consorciados: Benissa, Calpe, Dénia, Gata de Gorgos, Pedreguer, Pego, Teulada-Moraira, Xàbia.

- Elaboración de un inventario del patrimonio culinario de la Marina Alta a partir de la búsqueda y recopilación bibliográfica.

- Diseño de las herramientas informáticas y de difusión en las que se recopile este inventario.

- Diseño del catálogo del patrimonio culinario tradicional de la Marina Alta, que se ponga a disposición de la ciudadanía de la comarca.

El trabajo se centrará en dar cobertura a los siguientes principios:

- Investigación histórica. En tradición culinaria y alimentación local.

- Estructura y elementos de la gastronomía comarcal.

- Valorización. Estrategias de valorización territorial basadas en las potencialidades gastronómicas de productos agroalimentarios locales.

- Dinamización. Dinamización territorial y de turismo gastronómico, relacionado con los productos de la tierra.

- Divulgación y promoción. El conocimiento del patrimonio agroalimentario tradicional al alcance del público en general.

- Investigación y salud. Posibilidades de aplicación y valoración culinaria de productos concretos de nuestro patrimonio.

- Dimensión geográfica: el paisaje y los productos locales.

- Características climáticas mediterráneas 
- La singularidad de la tierra en la Marina Alta, origen de los productos que a su vez define tanto el entorno geográfico como el sentido gastronómico.

- Apuntes históricos. Referencias de historiadores sobre la tradición culinaria de la Marina Alta.

- Mapa y calendario gastronómico. Mapa de la comarca según sus productos agroalimentarios, puntos cardinales, fiestas y tradiciones culinarias locales.

\subsection{El patrimonio cultural gastronómico como Patrimonio Cultural Inmaterial}

La definición establecida por la Convención de la UNESCO considera Patrimonio Cultural Inmaterial "los usos, representaciones, expresiones, conocimientos y técnicas (junto con los instrumentos, objetos, artefactos y espacios culturales que les son inherentes) que las comunidades, los grupos y, en algunos casos los individuos reconozcan como parte integrante de su patrimonio cultural". En este sentido se enmarca el objetivo de identificar, difundir, promover y recuperar el patrimonio cultural gastronómico de la Marina Alta.

Los inventarios forman parte integrante de la salvaguardia del patrimonio cultural inmaterial porque pueden sensibilizar al público respecto de dicho patrimonio y de su importancia para las identidades individuales y colectivas

Además, el proceso de inventariar el patrimonio cultural inmaterial y poner los inventarios a disposición del público puede promover la creatividad y la autoestima de las comunidades y los individuos en los que se originan las expresiones y los usos de ese patrimonio.

Así, en la Carta sobre la preservación del patrimonio digital de UNESCO, define el carácter del patrimonio cultural como herencia común. El patrimonio digital consiste en recursos únicos que son fruto del saber o la expresión de los seres humanos. Comprende recursos de carácter cultural, educativo, científico o administrativo e información técnica, jurídica, médica y de otras clases, que se generan directamente en formato digital o se convierten a éste a partir de material analógico ya existente (...).

Muchos de esos recursos revisten valor e importancia duraderos, y constituyen por ello un patrimonio digno de protección y conservación en beneficio de las generaciones actuales y futuras. (...) El objetivo de la conservación del patrimonio digital es que éste sea accesible para el público.

Es preciso elaborar estrategias y políticas encaminadas a preservar el patrimonio digital, que tengan en cuenta el grado de urgencia, las circunstancias locales, los medios disponibles y las previsiones de futuro. La colaboración de los titulares de derechos de autor y derechos conexos y otras partes interesadas a la hora de definir formatos y compatibilidades comunes, así como el aprovechamiento compartido de recursos, pueden facilitar esa labor.

La propuesta de plan para confeccionar inventario de elementos del patrimonio cultural inmaterial, seguirán la línea marcada en este sentido por la UNESCO, esto es:

1. Identificación del elemento:

1.1. Nombre del elemento, tal como lo utiliza la comunidad o el grupo interesado.

1.2. Título breve y lo más informativo posible con indicación del (de los) ámbito(s).

1.3. Comunidad(es) concernida(s). 
1.4. Ubicación(es) física(s) del elemento.

1.5. Breve descripción.

2. Características del elemento:

2.1. Elementos materiales conexos.

2.2. Elementos inmateriales conexos.

2.3. Idioma(s), registro(s), nivel(es) de discurso.

2.4. Origen percibido.

3. Personas e instituciones relacionadas con el elemento:

3.1. Ejecutante(s)/intérprete(s).

3.2. Otros participantes.

3.3. Usos consuetudinarios que rigen el acceso al elemento o a algunos aspectos del mismo.

3.4. Modos de transmisión.

3.5. Organizaciones concernidas.

4. Estado del elemento: viabilidad:

4.1. Amenazas que pesan sobre la práctica.

4.2. Amenazas que pesan sobre la transmisión.

4.3. Disponibilidad de los elementos materiales y recursos conexos.

4.4. Viabilidad de los elementos materiales e inmateriales conexos.

4.5. Medidas de salvaguardia adoptadas.

5. Acopio e inventario de los datos:

5.1. Consentimiento de la comunidad o grupo al acopio e inventario de datos y participación en estas actividades.

5.2. Posibles restricciones del uso de los datos inventariados.

5.3. Experto(s): nombre y condición o pertenencia.

5.4. Fecha y lugar del acopio de datos.

5.5. Fecha de incorporación de los datos a un inventario.

5.6. Artículo del inventario compilado.

5.7. Referencias bibliográficas, discográficas, audiovisuales y archivísticas.

\section{IMPLEMENTACIÓN DEL PROYECTO}

El proyecto T'Avalem Marina Alta I se inicia en junio de 2017 una vez que CREAMA recibe la resolución de Labora (antiguo Servef) en la que se aprueba la realización de este proyecto con los recursos necesarios para llevarlo a cabo.

La realización del Inventario - Catálogo del patrimonio culinario de la Marina Alta en el marco de este proyecto se basa en procesos de innovación territorial y social en los que 
las redes territoriales, la cooperación institucional, la tradición, el "saber hacer" y los actores territoriales que han participado, han constituido elementos esenciales que han contribuido a su éxito, al mismo tiempo que el equipo docente de la rama administrativa y de gestión de la comunicación de este taller.

El diseño y determinación de la metodología se ha realizado mediante un procedimiento de debate y consenso por parte de un equipo multidisciplinar integrado por especialistas en diversas materias, todas ellas relacionadas con el territorio y su cultura.

Con el objetivo de que la recopilación se lleve a cabo mediante una disciplina rigurosa que permita realizar un trabajo de calidad, el 29 de junio de 2017 se constituye una comisión de seguimiento integrada por representantes de las entidades que ofrecen apoyo al programa, junto a representantes de la propia entidad y personal directivo y docente del proyecto. (MACMA, IECMA, Oficina de la Creativitat i la Innovació, CREAMA). Entre los integrantes de la comisión se cuenta con una persona especialista en archivística y documentación y otra persona en patrimonio cultural de la Marina Alta (Figura 1).

Figura 1. Composición de la Comisión de Seguimiento

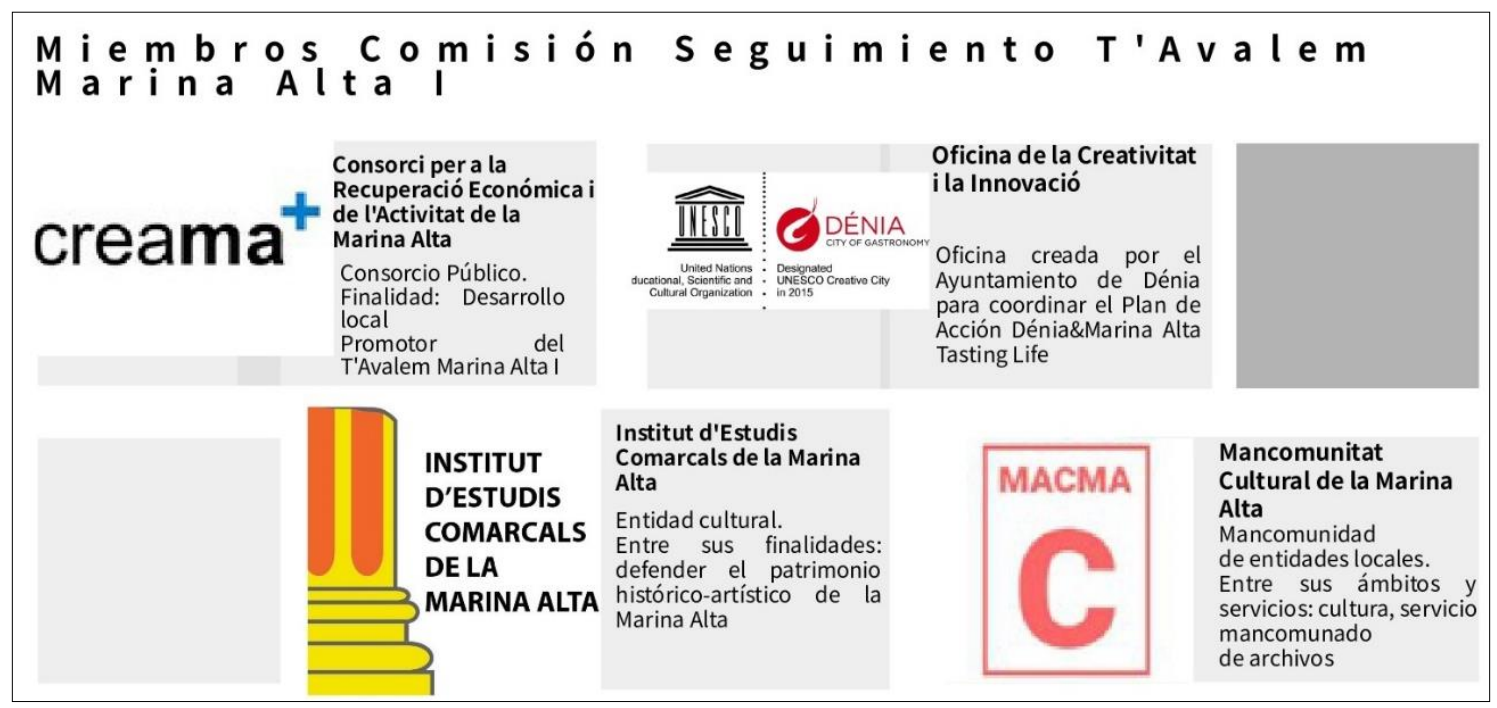

Fuente: elaboración propia.

El orden del día de esta primera reunión determina como objetivo de la misma la definición de los productos/resultados finales que va a elaborar el alumnado del Programa, así como la concreción del sistema de validación del proceso de elaboración.

Esta Comisión concreta tanto el alcance de la recopilación de datos, las fuentes con las que se inicia el trabajo, el contenido y formato de las fichas de recogida de datos y se analizan distintas opciones para el tratamiento digital de la información recogida.

En cuanto a la identificación de los materiales/documentos: la persona especializada en archivística plantea la propuesta consistente en la realización de un vaciado de toda la información que pueden contener los libros de fiestas de cada uno de los ocho municipios de CREAMA, en sus distintas festividades, y en cuanto al alcance se plantean las opciones siguientes: 
- Alcance temporal: iniciar el trabajo de inventariado desde el documento o material más antiguo, y en función de la magnitud de los documentos de cada municipio se compilará hasta una fecha durante la ejecución del proyecto T'Avalem.

- Alcance geográfico: ocho municipios que forman parte del consorcio: Benissa, Calp, Dénia, Gata de Gorgos, Pedreguer, Pego, Teulada y Xàbia.

En esta primera reunión se enfatiza sobre la importancia de recoger en una ficha única los datos que hagan posible la identificación y ubicación del documento/material para garantizar la utilidad, accesibilidad y localización del mismo una vez incorporado a la base de datos, así como sobre la importancia de prever un formato informático con el objeto de facilitar la accesibilidad y la incorporación contenido a las webs u otros soportes tecnológicos con los que cuenten las entidades que conforman la Comisión de Seguimiento y los municipios y entidades representadas. Se propone utilizar el formato de hoja de cálculo de Google o una plataforma online similar que permita crear, dar formato a hojas de cálculo y que permita la colaboración simultánea para garantizar la unidad, homogeneidad y actualización de la información y de los datos que se vayan introduciendo.

Con el objetivo de que el alumnado pueda realizar el trabajo utilizando la metodología consensuada por la Comisión de Seguimiento se acuerda impartir formación específica en materia de Patrimonio Histórico-Cultural de la Marina Alta para adquirir determinados conocimientos sobre clasificación de materiales y documentos, en aras a garantizar la fiabilidad, accesibilidad y localización de los mismos y completar la formación teórica relacionada con sistemas de archivo, así como información sobre el proyecto "Dénia, Ciudad Creativa de la UNESCO" para enmarcar el trabajo en su contexto. Además, se considera necesario que reciban formación sobre el uso del Google Docs, el programa gratuito basado en Web que permite crear documentos en línea con posibilidad de colaborar en grupo, disponible en el servicio de alojamiento de archivos Google Drive.

En la reunión de la Comisión de Seguimiento celebrada el 10/10/2017 se presenta la propuesta argumentada de ficha de recogida de datos elaborada por el alumnado. La Comisión tras incorporar pequeñas correcciones y matizaciones al documento, realiza la validación del mismo, aprobando el formato definitivo de la ficha (Figura 2).

La determinación tanto del alcance, como de la identificación del material/documentos a catalogar se decide también por consenso tras estudiar diferentes posibilidades, tomando el acuerdo de realizar el vaciado de la información de los libros de fiestas patronales desde el año 1990 en los ocho municipios que conforman CREAMA, especificándose que se extraerá cualquier contenido gastronómico que aparezca en los libros de fiestas patronales, con la siguiente clasificación: publicidad, artículos, recetas y actividades festeras (degustaciones, demostraciones y concursos), incorporándose en todo caso el detalle de la información a catalogar.

En la misma reunión se determina la fecha de la jornada formativa sobre la metodología a seguir en la realización del trabajo de prácticas objeto del proyecto. La jornada será impartida por la persona especializada en archivo y responsable del archivo municipal de Dénia, y por el periodista y asesor de comunicación de la Oficina de la Innovación y Creatividad de Dénia.

La siguiente sesión de la Comisión se celebra el 13 de diciembre de 2017, en la misma se informa del progreso de la catalogación, completada en estas fechas en un porcentaje de aproximadamente un $50 \%$ del alcance previsto. 
Figura 2. Ficha de recogida de datos

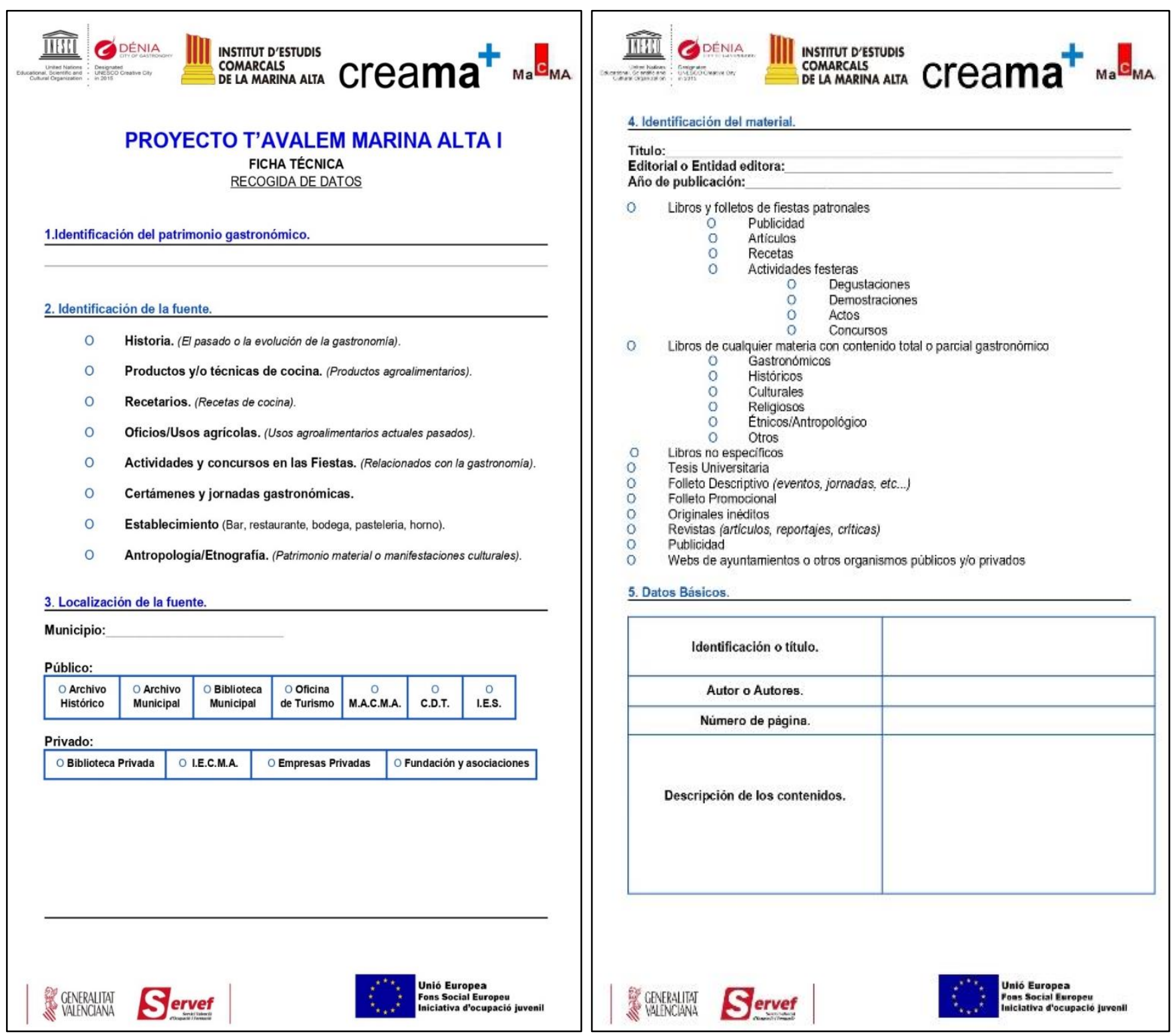

Fuente: T’Avalem Marina Alta I, elaboración propia.

Así mismo y con la finalidad de facilitar la utilización del catálogo se acuerdan los siguientes extremos:

- La realización de un listado unificado de las palabras clave que conformarán las búsquedas y que están contenidas en el apartado primero de la ficha con la denominación de Identificación del patrimonio gastronómico.

- Que la compilación de las palabras clave se realice sin tildes, a sugerencia de la especialista en archivística para facilitar las búsquedas informáticas en la base de datos.

- La incorporación de un anexo explicativo al listado de palabras clave que contenga notas aclaratorias con los criterios utilizados, todo ello al objeto de facilitar el trabajo de las personas que utilicen la base de datos para realizar búsquedas. Así tomando como ejemplo la palabra clave "Bar", en el anexo se especifica que ésta se ha utilizado para los establecimientos que elaboran comida como pizzería y pubs (Figura 3). 
En cuanto a la identificación de posibles búsquedas que se podrán realizar en el catálogo se articulan las siguientes:

- Búsqueda sencilla que incluya una palabra que quede localizada en cualquier campo de la ficha técnica. Ejemplo: arroz.

- Búsqueda avanzada que incluya dos o tres palabras con el filtro y/o: i) ejemplo 1: una palabra clave (mistela), en un año en concreto y por un autor (Antoni Reig); ii) ejemplo 2: una palabra clave, en una localidad y por fuentes (apartado 2 de la ficha técnica).

Figura 3. Palabra clave "Bar" - notas aclaratorias

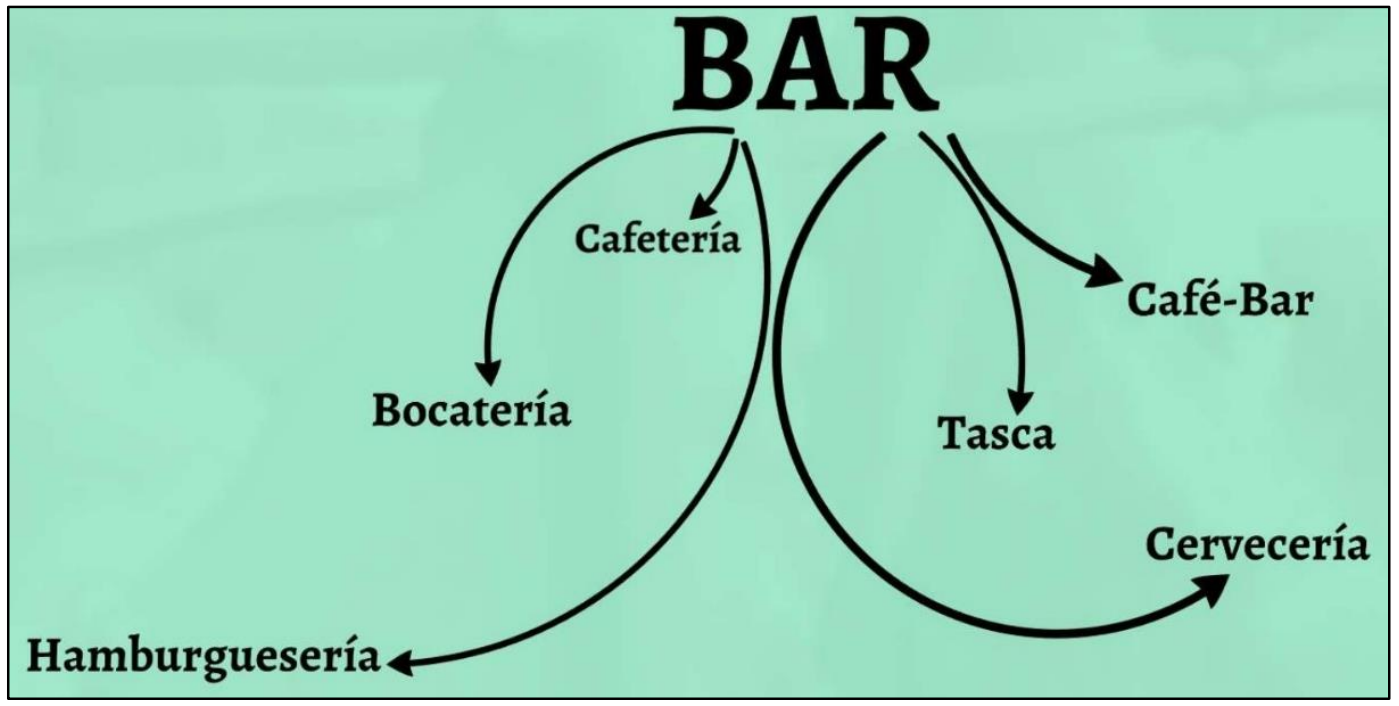

Fuente: T’Avalem Marina Alta I, elaboración propia.

La última reunión de la comisión de seguimiento se realiza el 07 de marzo de 2018, en la misma, la docente de la especialidad Susana Martínez Riutort, informa sobre el avance del trabajo, destacando el elevado número de fichas recogidas superando las 10.000, dando paso a la intervención de dos alumnas del programa que realizan una demostración de la base de datos creada, realizando varias búsquedas utilizando filtros sencillos (una condición) y filtros avanzados (usando de tres a cuatro condiciones). El objetivo de esta demostración es que la Comisión realice la validación conforme a la premisa de fiabilidad y utilidad.

En esta sesión se informa a los miembros de la comisión de que el programa informático para exportar la base de datos no es un gasto subvencionable por el proyecto y que sería conveniente buscar financiación alternativa para asumir dicho coste.

La presentación del resultado del trabajo de compilación realizado se expone en la clausura del Programa T'Avalem celebrada el día 29 de mayo de 2018.

Finalmente, el coste de la aplicación web para el almacenamiento y búsqueda y localización del inventario es asumido de forma compartida por las distintas agencias de desarrollo local que integran el Consorcio (Benissa, Calp, Dénia, Gata de Gorgos, Pedreguer, Pego, Teulada y Xàbia), incorporándose a la misma el contenido de un total de las 22.782 fichas de catalogación. 


\section{RESULTADOS}

Todo el proyecto está disponible en: http://www.marinaaltagastronomia.org/. El alcance logrado se sintetiza en la Figura 4. Las fichas de catalogación se recogen en la Tabla 1.

Figura 4. Alcance de la catalogación

\begin{tabular}{|c|c|c|}
\hline $\begin{array}{l}\text { LIBROS de } \\
\text { FIESTAS } \\
\text { PATRONALES }\end{array}$ & $\begin{array}{l}1990= \\
2017\end{array}$ & $=\frac{3}{3}$ \\
\hline Fuente & Temporal & Territorial \\
\hline \multicolumn{3}{|c|}{$\begin{array}{l}\text { - Datos gastronómicos históricos artículos. } \\
\text { - Actos festivos gastronómicos incluidos } \\
\text { programación fiestas. } \\
\text { - Anuncios relacionados con la gastronomía, como } \\
\text { bares, restaurantes, etc. }\end{array}$} \\
\hline
\end{tabular}

Fuente: T’Avalem Marina Alta I, elaboración propia.

Tabla 1. Fichas catalogadas por municipios

\begin{tabular}{|c|c|}
\hline Municipio & Núm. de fichas de catalogación \\
\hline Benissa & 1.771 \\
\hline Calp - Moros i Cristians & 2.166 \\
\hline Calp Festes Patronals Agost & 2.013 \\
\hline Dénia & 376 \\
\hline Dénia - Jesús Pobre & 1.462 \\
\hline Dénia - La Xara & 1.818 \\
\hline Gata de Gorgos & 1.807 \\
\hline Pedreguer & 2.151 \\
\hline Pego & 956 \\
\hline Teulada & 1.725 \\
\hline Teulada Moraira & 2.057 \\
\hline Xàbia - Fogueres & 1.632 \\
\hline Xàbia - Loreto & 1.958 \\
\hline Xàbia - Nazareno & 890 \\
\hline TOTAL & 22.782 \\
\hline
\end{tabular}

Fuente: T’Avalem Marina Alta I, elaboración propia.

Por último, la cronología del proyecto queda recogida en la Figura 5. 
Figura 5. Cronología del proyecto

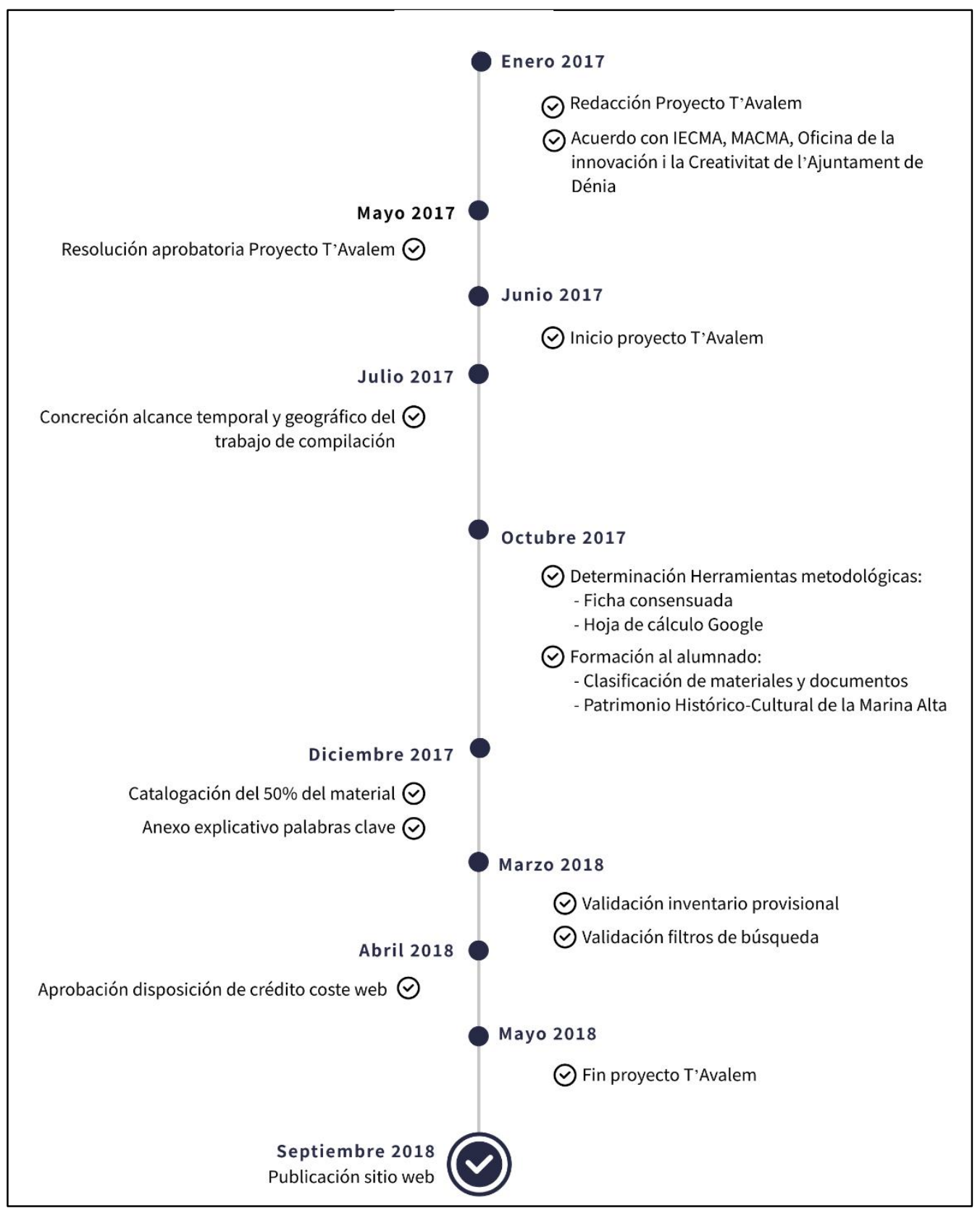

Fuente: T’Avalem Marina Alta I, elaboración propia.

\section{A UNIVERSITAT JAUME I}

Institut Interuniversitari de Desenvolupament Local

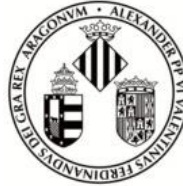

VNIVERSITAT i VAlÈnCia 